\title{
An Interactive Approach Based on Alternative Achievement Scale and Alternative Comprehensive Scale for Multiple Attribute Decision Making under Linguistic Environment
}

\author{
Yejun $\mathrm{Xu}^{1,2}$, Huimin Wang ${ }^{1,2^{*}}$, Daniel Palacios-Marqués ${ }^{3}$ \\ ${ }^{1}$ State Key Laboratory of Hydrology-Water Resources and Hydraulic Engineering, Hohai University, Nanjing, 210098, \\ PR China \\ ${ }^{2}$ Research Institute of Management Science, Business School, Hohai University, Nanjing, 211100, PR China \\ ${ }^{3}$ Department of Business Administration, Camino Vera s/n CP: 46022, Universitat Politècnica Valencia, Spain
}

Received 21 February 2011

Accepted 14 october 2012

\begin{abstract}
The aim of this paper is to develop an interactive approach for multiple attribute decision making with incomplete weight information under linguistic environment. Some of the concepts are defined, such as the distance between two 2-tuple linguistic variables, the expectation level of alternative, the achievement scale, the alternative comprehensive scale under linguistic environment. Based on these concepts, we establish some linear programming models, through which the decision maker interacts with the analyst. Furthermore, we establish a practical interactive approach for selecting the most desirable alternative(s). The interactive process can be realized by giving and revising the achievement scale and comprehensive scale of alternatives till the achievement scale and the comprehensive scale are achieved to the decision maker's request. Finally, an illustrative example is also given.
\end{abstract}

Keywords: Multiple attribute decision making; Interactive approach; Achievement scale; Comprehensive scale; 2tuple linguistic.

\section{Introduction}

Multiple attribute decision making (MADM) consists of selecting the most desirable alternative from a given alternative set. Usually, it consists the following two phases ${ }^{3,6,20}$ : (1) Aggregation phase: it consists of establishing an appropriate aggregation operator for aggregating and combining the individual preferences values provided from decision maker to obtain a collective preference value for each alternative; (2) Exploitation phase: it consists of establishing a rank ordering among the alternatives according to the collective preference value for choosing the best alternative(s). For the above processes, the selection process is only made in a single step, that means each phase is only implemented once. But in the real world, the increasing complexity of the socio-economic environment makes it less and less impossible for the decision maker (DM) to make a decision in an order step, perhaps in some phase, the DM does not satisfy with the result, it needs to revise some value of the phase, thus, interactive process is important for the DM to determine the best alternative. Interactive approaches have been received more and more attention in recent years ${ }^{2,11-14,19,31,32}$. As a result, the above methods can only be suitable for dealing with the MADM problems with numerical information on attributes values. In the real-life word, there are many

* Corresponding author: hmwang@hhu.edu.cn 
decision situations in which the information cannot be assessed by precisely in a quantitative form but may be in a qualitative one, for example, when evaluating the "comfort" or "design" of a car, terms like "good", "medium", "bad" are usually used, and evaluating a car's speed, terms like "very fast”, "fast”, "slow” can be

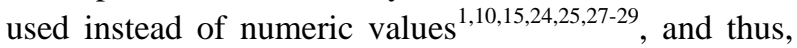
in such situations, the use of linguistic approach is necessary. $\mathrm{Xu}^{30}$ developed an interactive approach to multiple attribute group decision making with multigranular uncertain linguistic information. $\mathrm{Xu}^{32}$ presented an interactive procedure for linguistic multiple attribute decision making with incomplete weight information. The method establishes a practical interactive procedure for selecting the most desirable alternative(s). The interactive process can be realized by giving and revising the satisfactory degrees of alternatives till an optimum satisfactory solution is achieved. But in the paper, the satisfactory degree of alternative is so simple, and far away from the real situations. And in fact, in the process of the interactive, the satisfactory degree of alternative, in our sense, is a subjective index, and only expressed by a numerical value, it is not only difficult, but also deprives the DM's leading role to some extent. All of the papers are failed to deal with such situations. Therefore, it is important to pay attention to this issue. And also $\mathrm{Xu}^{32}$ used the virtual linguistic variable to compute. However, Martínez et al. ${ }^{17}$ said that the appearance of virtual terms without syntax either semantics limits the interpretability of the results. The 2-tuple linguistic model is a symbolic computational one introduced by Herrera and Martínez ${ }^{8}$ in order to improve the accuracy and facilitate the processes of CW $18,35,36$ by treating the linguistic domain as continuous but keeping the linguistic basis (syntax and semantics). The 2-tuple has been discussed extensively ${ }^{5-7,9,16,26}$ in the last years and also has a new version ${ }^{4,21}$. Therefore, we use 2-tuple linguistic model to deal with linguistic information in this paper.

In this paper, we focus our attention on developing a practical interactive procedure for selecting the most desired alternative(s) by 2-tuple linguistic model under linguistic environment. We investigate the MADM problems, in which the information about attribute weights is partly known and the attribute values are expressed by means of linguistic variables. We propose the objective index of achievement scale. It depends on the DM to judge the satisfactory degree of alternative according to the achievement scale. It not only avoids expressing the satisfactory degree by a simple index, but also maintains the DM's leading role. In order to measure the overall value of all the alternatives, we introduce the comprehensive scale. We don't need the index of comprehensive scale to reflect the overall value precisely, but it provides the DM to know the overall value in the interactive decision process. The interactive process can be realized by giving and revising the achievement scale and comprehensive scale till an optimum satisfactory solution is achieved.

In order to do this, this paper is structured as follows. Section 2 gives the concept of the 2-tuple linguistic representation, the distance between two 2tuple linguistic variables. Section 3 defines the expectation level of alternative, the 2-tuple linguistic negative point, the achievement scale, the alternative comprehensive scale under linguistic environment. Based on these concepts, an interactive approach is developed. Section 4 , a practical application of the developed approach to evaluate the university faculty for tenure and promotion has also been given to show the effectiveness of the proposed approach. Section 5, concludes the paper and points out the future work.

\section{Preliminaries}

The linguistic variables are used in processes of computing with words that imply their fusion, aggregation, comparison, etc. The most often used models dealing with linguistic information are: (i ) the semantic model that uses the linguistic terms just as labels for fuzzy numbers, while the computations over them are done directly over those fuzzy numbers; (ii ) the second one, is the symbolic model that uses the order index of the linguistic terms to make direct computation on labels; (iii) the third mode is based on the linguistic 2-tuple.

The 2-tuple linguistic representation model presented in Ref. 8,9, is based on the symbolic one and in a concept called Symbolic Translation. In this section, we shall make a brief review of some concepts of the 2tuple.

Definition 1. Let $S=\left\{s_{0}, s_{1}, \ldots, s_{g}\right\}$ be a linguistic term set. For example, a set of nine terms $S$ could be given as follows:

$$
\begin{aligned}
& S=\left\{s_{0}=\text { extremely poor }, s_{1}=\text { very poor }, s_{2}=\text { poor },\right. \\
& S_{3}=\text { slightly poor }, s_{4}=\text { fair }, s_{5}=\text { slightly good }, \\
& \left.s_{6}=\text { good , } s_{7}=\text { very good }, s_{8}=\text { extremely good }\right\}
\end{aligned}
$$


Usually, in these cases, it is required that the linguistic term set satisfies the following additional characteristics.

1) There is a negation operator: $\operatorname{Neg}\left(s_{i}\right)=s_{j}$ such that $j=g-i(g+1$ is the cardinality).

2) $s_{i} \leq s_{j} \Leftrightarrow i \leq j$. Therefore, there exists a minimization and a maximization operator.

Definition $2^{8}$. Let $\beta$ be the result of an aggregation of the indexes of a set of labels assessed in a linguistic term set $S$, i.e., the result of a symbolic aggregation operation. $\beta \in[0, g]$, being $g$ the cardinality of $S$. Let $i=$ $\operatorname{round}(\beta)$ and $\alpha=\beta$-i be two values such that $i \in[0, g]$ and $\alpha \in[-0.5,0.5]$, then $\alpha$ is called a symbolic translation.

From this concept it is developed a linguistic representation model which represents the linguistic information by means of 2-tuple $\left(s_{i}, \alpha_{i}\right), s_{i} \in S$ and $\alpha_{i} \in[-$ 0.5,0.5]:

- $s_{i}$ represents the linguistic label center of the information;

- $\alpha_{i}$ is a numerical value expressing the value of the translation from the original result $\beta$ to the closest index label $i$ in the linguistic term set $\left(s_{i}\right)$, i.e., the symbolic translation.

Definition $3^{8}$. Let $S=\left\{s_{0}, s_{1}, \ldots, s_{g}\right\}$ be a linguistic term set and $\beta \in[0, g]$ be a value representing the result of $a$ symbolic aggregation operation, then the 2-tuple that expresses the equivalent information to $\beta$ is obtained with the following function:

$$
\begin{aligned}
& \Delta:[0, g] \rightarrow S \times[-0.5,0.5) \\
& \Delta(\beta)=\left(s_{i}, \alpha_{i}\right), \text { with }\left\{\begin{array}{l}
s_{i}, i=\operatorname{round}(\beta) \\
\alpha_{i}=\beta-i, \alpha_{i} \in[-0.5,0.5)
\end{array}\right.
\end{aligned}
$$

where round $(\cdot)$ is the usual round operation, $s_{i}$ has the closest index label to $\beta$ and $\alpha_{i}$ is the value of the symbolic translation.

Contrarily, let $S=\left\{s_{0}, S_{1}, \ldots, S_{g}\right\}$ be a linguistic term set and $\left(s_{i}, \alpha_{i}\right)$ be a 2 -tuple. There is always a $\Delta^{-1}$ function:

$$
\begin{aligned}
& \Delta^{-1}: S \times[-0.5,0.5) \rightarrow[0, g] \\
& \Delta^{-1}\left(s_{i}, \alpha_{i}\right)=i+\alpha_{i}=\beta
\end{aligned}
$$

Remark: From Definitions 2 and 3, it is obvious that the conversion of a linguistic term into a linguistic 2tuple consists of adding a value 0 as symbolic translation:

$$
s_{i} \in S \Rightarrow\left(s_{i}, 0\right) .
$$

Comparison of 2-Tuples: let $\left(s_{k}, \alpha_{k}\right)$ and $\left(s_{l}, \alpha_{l}\right)$ be two 2-tuples, with each one representing a counting of information as follows:

1) If $k<l$ then $\left(s_{k}, \alpha_{k}\right)<\left(s_{l}, \alpha_{l}\right)$;

2) If $k=l$ (1) $\alpha_{k}=\alpha_{l}$ then $\left(s_{k}, \alpha_{k}\right)=\left(s_{l}, \alpha_{l}\right)$;

(2) $\alpha_{k}<\alpha_{l}$ then $\left(s_{k}, \alpha_{k}\right)<\left(s_{l}, \alpha_{l}\right)$;
(3) $\alpha_{k}>\alpha_{l}$ then $\left(s_{k}, \alpha_{k}\right)>\left(s_{l}, \alpha_{l}\right)$.

For a multiple attribute decision making problem, let $X=\left\{x_{1}, x_{2}, \ldots, x_{n}\right\}$ be a discrete set of alternatives, $U=\left\{u_{1}\right.$, $\left.u_{2}, \ldots, u_{m}\right\}$ be a set of attributes. For each alternative $x_{j} \in X$, the decision maker gives his/her preference value $a_{i j}$ with respect to attribute $u_{i} \in U$, where $a_{i j}$ takes the form of linguistic variable, that is $a_{i j} \in S$, then all the preference values of the alternatives consist of the decision matrix $A=\left(a_{i j}\right)_{m \times n}$.

Definition 4. Let $\left\{\left(r_{1}, \alpha_{1}\right),\left(r_{2}, \alpha_{2}\right) \ldots,\left(r_{m}, \alpha_{m}\right)\right\} \quad\left(r_{i} \in S, \alpha_{i} \in[-\right.$ $0.5,0.5], i=1,2, \ldots, m)$ be a set of linguistic 2-tuples,

then 2TLWA is called 2-tuple linguistic weighted averaging (2TLWA) operator, if

$$
2 \operatorname{TLWA}\left(\left(r_{1}, \alpha_{1}\right), \ldots,\left(r_{m}, \alpha_{m}\right)\right)=\Delta\left(\frac{\sum_{i=1}^{m} w_{i} \Delta^{-1}\left(r_{i}, \alpha_{i}\right)}{\sum_{i=1}^{m} w_{i}}\right)
$$

where $w=\left(w_{1}, w_{2}, \ldots, w_{m}\right)^{T}$ is the weighting vector of the 2-tuples $\left(r_{i}, \alpha_{i}\right)(i=1,2, \ldots, m)$. Generally, $\sum_{i=1}^{m} w_{i}=1, w_{i} \in$ $[0,1], \quad i=1,2, \ldots, m$. Thus, Eq.(1) is reduced to the following:

$$
2 \operatorname{TLWA}\left(\left(r_{1}, \alpha_{1}\right), \ldots,\left(r_{m}, \alpha_{m}\right)\right)=\Delta\left(\sum_{i=1}^{m} w_{i} \Delta^{-1}\left(r_{i}, \alpha_{i}\right)\right)
$$

Especially, if $w=(1 / m, 1 / m \ldots, 1 / m)^{T}$, then the $2 T L W A$ operator is reduced to the 2-tuple linguistic average (2TLA) operator.

Definition $5^{8}$. Let $A=\left(a_{i j}\right)_{m \times n}$ be the linguistic decision matrix, $a_{j}=\left(a_{1 j}, a_{2 j}, \ldots, a_{m j}\right)$ be the vector of attribute values corresponding to the alternative $x_{j}, j=1,2, \ldots, n$, then we call

$z_{j}(w)=2 \operatorname{TLWA}_{w}\left(a_{1 j}, a_{2 j}, \ldots, a_{m j}\right)=\Delta\left(\sum_{i=1}^{m} w_{i} \Delta^{-1}\left(a_{i j}\right)\right)$

the overall value of the alternative $x_{j}$, where $w=\left(w_{1}\right.$, $\left.w_{2}, \ldots, w_{m}\right)^{T}$ is the weighting vector of attributes.

The linguistic multiple attribute decision making problems generally consist of finding the most desirable alternative(s) from a given alternative set. If the decision matrix $A=\left(a_{i j}\right)_{m \times n}$ and the corresponding weight value of attributes are known, we can get the overall value of the alternative $x_{j}$ by Eq.(3).

Definition 6. Let $\left(r_{k}, \alpha_{k}\right)$ and $\left(r_{l}, \alpha_{l}\right)$ be two 2-tuples, then we call

$$
d\left(\left(r_{k}, \alpha_{k}\right),\left(r_{l}, \alpha_{l}\right)\right)=\left|k+\alpha_{k}-l-\alpha_{l}\right|
$$

the distance between $\left(r_{k}, \alpha_{k}\right)$ and $\left(r_{l}, \alpha_{l}\right)$.

Obviously, the smaller the value of $d\left(\left(r_{k}, \alpha_{k}\right),\left(r_{l}, \alpha_{l}\right)\right)$, the closer $\left(r_{k}, \alpha_{k}\right)$ to $\left(r_{l}, \alpha_{l}\right)$, thus, $d\left(\left(r_{k}, \alpha_{k}\right),\left(r_{l}, \alpha_{l}\right)\right)$ can be used as a deviation measure of two 2-tuples and it is very 
easy to compute. The distance between $\left(r_{k}, \alpha_{k}\right)$ and $\left(r_{l}, \alpha_{l}\right)$ is more intuitive .

In the real world, the information about attribute weights is incompletely known. Let $w=\left(w_{1}, w_{2}, \ldots, w_{m}\right)^{T}$ $\in H$ be the weight vector of attributes, where $w_{i} \in[0$, 1], $i=1,2, \ldots, m, \sum_{i=1}^{m} w_{i}=1, H$ is a set of the known weight information, which can be constructed by the following forms ${ }^{12-14,19,23,32,33}$, for $i \neq j$.

Form1. A weak ranking: $\left\{w_{i} \geq w_{j}\right\}$;

Form2. A strict ranking: $\left\{w_{i}-w_{j} \geq \alpha_{i}(>0)\right\}$;

Form3. A ranking of differences: $\left\{w_{i}-w_{j} \geq w_{k}-w_{l}\right\}$, for $j \neq k \neq l$;

Form4. A ranking with multiples: $\left\{w_{i} \geq \alpha_{i} w_{j}\right\}, 0 \leq \alpha_{i}$ $\leq 1$

Form5. An interval form: $\alpha_{i} \leq w_{i} \leq \alpha_{i}+\varepsilon_{i}, 0 \leq \alpha_{i}<$ $\alpha_{i}+\varepsilon_{i} \leq 1$.

Forms 1-2 and Forms 4-5 are well known types of imprecise information, and Form 3 is ranking of differences of adjacent parameters obtained by ranking between two parameters, which can be constructed based on Form1.

\section{Models and Approach}

Motivated the ideal by Ref. 31 and 32, we define the following concepts, which will be used in the following sections.

Definition 7. The overall $z_{j}^{*}$ value of alternative $x_{j}$ which the decision maker expects to achieve is called the expectation level of alternative $x_{j}$.

Definition $8^{32}$. Let $a_{i}^{-}=s_{0}=\left(s_{0}, 0\right)$, for all $j$, then we call $a^{-}=\left(a_{1}^{-}, a_{2}^{-}, \ldots, a_{m}^{-}\right)$the 2-tuple linguistic negative ideal point.

Especially, we get the overall value corresponding to the linguistic negative ideal point as follows,

$$
z^{-}(w)=\Delta\left(\sum_{i=1}^{m} w_{i} \Delta^{-1}\left(s_{0}, 0\right)\right)=s_{0}
$$

Definition 9. Let $f\left(z_{j}(w)\right)=d\left(z_{j}(w), z^{-}(w)\right)$ be the distance between the overall value $z_{j}(w)$ of the alternative $x_{j}$ and the overall value $z^{-}(w)$ corresponding to the linguistic negative point $a^{-}$.

Obviously, the greater the distance between the overall value $z_{j}(w)$ of the alternative $x_{j}$ and the overall value $z^{-}(w)$ corresponding to the linguistic negative point $a^{-}$, the higher value of $f\left(z_{j}(w)\right)$, that is, the value $f\left(z_{j}(w)\right)$ of the alternative $x_{j}$ is a strictly monotone increasing function. The higher $f\left(z_{j}(w)\right)$, the better the alternative $x_{j}$ is. As a result, we establish the following optimization model:
(M-1)

$\max f(w)=\left(f\left(z_{1}(w), f\left(z_{2}(w)\right), \ldots, f\left(z_{j}(w)\right)\right)\right.$

s.t. $w \in H$, $\sum_{i=1}^{m} w_{i}=1, w_{i} \geq 0, i=1,2, \ldots, m$.

Definition $10^{32}$. Let $d\left(z_{j}^{*}, z^{-}(w)\right)$ be the distance between the expectation level of alternative $x_{j}$ and the overall value $z^{-}(w)$ corresponding to the linguistic negative ideal point $a^{-}$, then we call

$$
\mu\left(z_{j}(w)\right)=\frac{d\left(z_{j}(w), z^{-}(w)\right)}{d\left(z_{j}^{*}, z^{-}(w)\right)}
$$

the achievement scale of the alternative $x_{j}$.

Obviously, function $\mu\left(z_{j}(w)\right)$ has the following features:

(1) the alternative achievement scale is that the percentage of the achieved overall value of alternative to the expectation level, which is based on the premise of the minimum of the overall value. If the overall value $z_{j}(w)$ farther way from the minimum, the achievement scale of alternative is higher, otherwise, the achievement scale of alternative is lower.

(2) for $w^{\prime}, w^{\prime \prime} \in H$, if $d\left(z_{j}\left(w^{\prime}\right), z^{-}\left(w^{\prime}\right)\right)>d\left(z_{j}\left(w^{\prime \prime}\right)\right.$, $\left.z^{-}\left(w^{\prime \prime}\right)\right)$, then $\mu\left(z_{j}\left(w^{\prime}\right)\right)>\mu\left(z_{j}\left(w^{\prime \prime}\right)\right)$, that is, $\mu\left(z_{j}(w)\right)$ is a strictly monotone increasing function on $z_{j}(w)$.

Definition 11. Let $c(w)=\sum_{j=1}^{n} d\left(z_{j}(w), z^{-}(w)\right)$, then $c(w)$ is called the alternative comprehensive scale.

The alternative achievement scale denotes the situation of each alternative achieved. The alternative comprehensive scale denotes the overall value of all alternatives. Taking into account the above two faces, then, in the interactive decision process, we could not only achieve the decision maker's request in the overall value of all alternatives, but could lead each alternative to achieve its better status as possible, and thus rank the alternative reasonably. The main idea is following. In the first, let the alternative comprehensive scale achieve the maximum, which is to construct the following single objective model:

(M-2) $\quad \max c(w)$

$$
\begin{array}{ll}
\text { s.t. } & w \in H \\
& \sum_{i=1}^{m} w_{i}=1, w_{i} \geq 0, i=1,2, \ldots, m .
\end{array}
$$

Solving this model, we get the best solution $w^{(0)}$, the overall value of each alternative $z_{j}\left(w^{(0)}\right)$ and the comprehensive scale $c\left(w^{(0)}\right)$, and also we get the achievement scale $\mu\left(z_{j}\left(w^{(0)}\right)\right)(j=1,2, \ldots, n)$. In the course of decision making, the decision maker then provides 
the initial lower bounds $\lambda_{j}^{(0)}(j=1,2, \ldots, n)$ of the achievement scale of the alternatives $x_{j}(j=1,2, \ldots, n)$ according to $\mu\left(z_{j}\left(w^{(0)}\right)\right)$ and the lower bound $c_{0}$ of the alternative comprehensive scale.

The better of the alternative comprehensive scale, the more satisfies to the decision maker's request in the whole of all the alternatives. But it will be lower the achievement scale of some alternatives, and thus far way from its better status. On the other hand, if we only utilize the achievement scale, it could not realize the equivalent of different alternatives effectively, and also all the alternatives are fairly competitive. As a result, we establish the following optimization model:

(M-3)

$$
\max J=\sum_{j=1}^{n} \lambda_{j}
$$

$$
\begin{array}{ll}
\text { s.t. } & c(w) \geq c_{0} \\
& \mu\left(z_{j}(w)\right) \geq \lambda_{j} \geq \lambda_{j}^{(0)}, \\
& w \in H, \sum_{i=1}^{m} w_{i}=1, w_{i} \geq 0, i=1,2, \ldots, m .
\end{array}
$$

Solving the model (M-3), if there exists no optimal solution, then the decision maker needs to reconsider the lower bounds $c_{0}$ of the comprehensive scale and the lower bounds $\lambda_{j}^{(0)}(j=1,2, \ldots, n)$ of the achievement scale of the alternatives $x_{j}(j=1,2, \ldots, n)$ till the optimal solution is obtained.

Theorem 1. The optimal solution of the model (M-2) is the Pareto solution of the model (M-1).

Proof. Suppose that $w^{*}$ is the optimal solution of the model (M-2), and $w^{*}$ is not the Pareto solution of the model (M-1), then there exists $w^{\prime}$ such that $f\left(z_{j}\left(w^{\prime}\right)\right)>$ $f\left(z_{j}\left(w^{*}\right)\right)$, for all $x_{j} \in X$, and there exists $x_{j_{0}} \in X$, such that $f\left(z_{j_{0}}\left(w^{\prime}\right)\right)>f\left(z_{j_{0}}\left(w^{*}\right)\right)$. As $c(w)$ is a strictly monotone increasing function on $f\left(z_{j}(w)\right)$, thus

$$
c\left(w^{\prime}\right)>c\left(w^{*}\right)
$$

which contradicts to the supposition condition. This completes proof of Theorem 1 .

Theorem 2 . The optimal solution of the model (M-3) is the Pareto solution of the model (M-1).

Proof. Suppose that $w^{*}$ is the optimal solution of the model (M-3), and $w^{*}$ is not the Pareto solution of the model (M-1), then there exists $w^{\prime}$ such that $f\left(z_{j}\left(w^{\prime}\right)\right)>$ $f\left(z_{j}\left(w^{*}\right)\right)$, for all $x_{j} \in X$, and there exists $x_{j_{0}} \in X$, such that $f\left(z_{j_{0}}\left(w^{\prime}\right)\right)>f\left(z_{j_{0}}\left(w^{*}\right)\right)$. Because $\mu\left(z_{j}(w)\right)$ is also a strictly monotone increasing function on $f\left(z_{j}(w)\right)$, so $\mu\left(z_{j_{0}}\left(w^{\prime}\right)\right)>\mu\left(z_{j_{0}}\left(w^{*}\right)\right)$. Then $\mu\left(z_{j}\left(w^{\prime \prime}\right)\right) \geq \lambda_{j} \geq \lambda_{j}^{(0)}$, for all $x_{j} \in X$, and there exists $\lambda_{j_{0}}^{\prime}$, such that $\mu\left(z_{i}\left(w^{\prime \prime}\right)\right)>$ $\lambda_{j_{0}}^{\prime}>\lambda_{j_{0}}>\lambda_{j}^{(0)}$. Thus

$$
\sum_{j=1, j \neq j_{0}}^{n} \lambda_{j}+\lambda_{j_{0}}^{\prime}>\sum_{j=1}^{n} \lambda_{j}
$$

which contradicts to the supposition condition. This completes proof of Theorem 2 .

Theorem 1 and Theorem 2 denote that the optimal solution of the model (M-2) and (M-3) is the Pareto solution of the model (M-1). If the decision maker satisfies the optimal solution of (M-3), then we can utilize the Eq.(3) to get the overall value $z_{j}(w) \quad(j=1$, $2, \ldots, n)$ of each alternative. Then we rank $z_{j}(w) \quad(j=1$, $2, \ldots, n)$ in descending order.

Based on the above models and theorems, we develop a practical method for solving the linguistic multiple attribute decision making problems, in which the information about attribute weights is incompletely known, and the attribute values take the form of linguistic variables, and the decision procedure need the decision maker to modify his/her request constantly until the decision maker satisfies the result. The method involves the following steps:

Step1. Let $X=\left\{x_{1}, x_{2}, \ldots, x_{n}\right\}$ be a discrete set of alternatives, $U=\left\{u_{1}, u_{2}, \ldots, u_{m}\right\}$ be a set of attributes, and $w=\left(w_{1}, w_{2}, \ldots, w_{m}\right)^{T} \in H$ be the weight vector of attributes, where $\sum_{i=1}^{m} w_{i}=1, w_{i} \in[0,1], i=1,2$, $\ldots, m, H$ is a set of the known weight information, which can be constructed by the Forms 1-5. For each alternative $x_{j} \in X$, the decision maker gives his/her preference value $a_{i j}$ with respect to attribute $u_{i} \in U$, where $a_{i j}$ takes the form of linguistic variable, that is $a_{i j}$ $\in S$, then all the preference values of the alternatives consist of the decision matrix $A=\left(a_{i j}\right)_{m \times n}$. The decision maker gives his/her expectation level $z_{j}^{*}$ of each alternative. Let $k^{*}$ be the maximum iteration number, and $k=0$.

Step 2. Utilize the model (M-2) to derive the original optimal solution $w^{(0)}=\left(w_{1}^{(0)}, w_{2}^{(0)}, \ldots, w_{m}^{(0)}\right)^{T}$, then calculate the alternative comprehensive scale $c\left(w^{(0)}\right)$, and also calculate the achievement scale $\mu\left(z_{j}\left(w^{(0)}\right)\right)(j=1,2, \ldots, n)$. The decision maker gives the lower bounds $\lambda_{j}^{(0)}(j=1,2, \ldots, n)$ of the achievement scale of the alternatives $x_{j}(j=1,2, \ldots, n)$ according to the achievement scale $\mu\left(z_{j}\left(w^{(0)}\right)\right)(j=$ 
$1,2, \ldots, n)$, and also gives the lower bound $c_{0}$ of the alternative comprehensive scale. Let $k=1$.

Step 3. Utilize the model (M-3) to derive the weight vector $w^{(k)}=\left(w_{1}^{(k)}, w_{2}^{(k)}, \ldots, w_{m}^{(k)}\right)^{T}$ and calculate the alternative comprehensive scale $c\left(w^{(k)}\right)$, and also calculate the achievement scale $\mu\left(z_{j}\left(w^{(k)}\right)\right)$ $(j=1,2, \ldots, n)$.

Step 4. If the decision maker is satisfied with the result obtained by Step 3 or $k=k^{*}$, then calculate the overall values $z_{j}\left(w^{(k)}\right)(j=1,2, \ldots, n)$ of the alternatives $x_{j}(j=1,2, \ldots, n)$ by using Eq.(3), and rank all alternatives according to the values of $z_{j}\left(w^{(k)}\right)$ $(j=1,2, \ldots, n)$ in descending order, and then go to Step 5; if there exists no solution for the model (M-3) or the result does not satisfy the decision maker, then the decision maker should increase the achievement scale of some alternatives, and decrease the achievement scale of some alternatives, and also if necessary, adjust the lower bound of the comprehensive scale $c_{k}$. Let $k=k+1$, and return to Step 3 .

\section{Step 5. End.}

\section{Illustrative Example}

In this section, a MADM problem of evaluating university faculty for tenure and promotion is used to illustrate the developed procedure. The attributes used at some universities are (adapted from $\mathrm{Xu}^{32}$ ): $u_{1}$ : teaching, $u_{2}$ : research, and $u_{3}$ :service. Five faculty candidates (alternatives) $x_{j}(j=1,2,3,4,5)$ are evaluated using the linguistic label set

$S=\left\{s_{0}=\right.$ extremely poor , $s_{1}=$ very poor , $s_{2}=$ poor ,

$s_{3}=$ slightly poor,$s_{4}=$ fair,$s_{5}=$ slightly good,

$s_{6}=\operatorname{good}, s_{7}=$ very good,$s_{8}=$ extremely good $\}$

by the decision maker under these three attributes, as listed in Table 1.

Suppose that the known weight information is as follows:

$$
H=\left\{0.25 \leq w_{1} \leq 0.4,0.15 \leq w_{2} \leq 0.3, w_{3}>w_{2}\right\}
$$

To get the most desirable alternative(s), the following steps are involved:

Table 1. Linguistic decision matrix $A$

\begin{tabular}{llllll}
\hline$u_{i}$ & $x_{1}$ & $x_{2}$ & $x_{3}$ & $x_{4}$ & $x_{5}$ \\
\hline$u_{1}$ & $\mathrm{~s}_{6}$ & $\mathrm{~s}_{7}$ & $\mathrm{~s}_{4}$ & $\mathrm{~s}_{8}$ & $\mathrm{~s}_{6}$ \\
$u_{2}$ & $\mathrm{~s}_{7}$ & $\mathrm{~s}_{8}$ & $\mathrm{~s}_{7}$ & $\mathrm{~s}_{3}$ & $\mathrm{~s}_{7}$ \\
$u_{3}$ & $\mathrm{~s}_{4}$ & $\mathrm{~s}_{2}$ & $\mathrm{~s}_{6}$ & $\mathrm{~s}_{6}$ & $\mathrm{~s}_{5}$ \\
\hline
\end{tabular}

Step 1. The decision maker gives his expectation level $z_{j}^{*}(j=1,2, \ldots, 5)$ of each alternative $x_{j}(j=1,2, \ldots, 5)$ as follows:

$$
\begin{aligned}
& z_{1}^{*}=\left(s_{6}, 0.2\right), z_{2}^{*}=\left(s_{7},-0.5\right), z_{3}^{*}=\left(s_{6}, 0\right), \\
& z_{4}^{*}=\left(s_{6}, 0.4\right), z_{5}^{*}=\left(s_{7},-0.4\right) .
\end{aligned}
$$

Step 2. Utilize the model (M-2) to establish the following optimization model:

$$
\max 11 w_{1}+12 w_{2}+3 w_{3}+20
$$

s.t. $0.25 \leq w_{1} \leq 0.4,0.15 \leq w_{2} \leq 0.3, w_{3}>w_{2}$,

$$
w_{1}+w_{2}+w_{3}=1, w_{j} \geq 0, j=1,2,3 \text {. }
$$

Solving this model, we obtain the original optimal solution:

$$
w^{(0)}=(0.4,0.3,0.3)^{T}
$$

and calculate the alternative comprehensive scale $c\left(w^{(0)}\right)$, and also calculate the achievement scale $\mu\left(z_{j}\left(w^{(0)}\right)\right)(j=1,2, \ldots, 5)$.

$$
\begin{aligned}
& c\left(w^{(0)}\right)=28.9, \mu\left(z_{1}\left(w^{(0)}\right)\right)=0.9194, \\
& \mu\left(z_{2}\left(w^{(0)}\right)\right)=0.8923, \mu\left(z_{3}\left(w^{(0)}\right)\right)=0.9167, \\
& \mu\left(z_{4}\left(w^{(0)}\right)\right)=0.9219, \mu\left(z_{5}\left(w^{(0)}\right)\right)=0.9091 .
\end{aligned}
$$

The decision maker gives the lower bounds $\lambda_{j}^{(0)}$ $(j=1,2, \ldots, 5)$ of the achievement scale of the alternatives $x_{j}(j=1,2, \ldots, 5)$ according to the achievement scale $\mu\left(z_{j}\left(w^{(0)}\right)\right)(j=1,2, \ldots, 5)$, and gives the lower bound of the comprehensive scale $c_{0}$ as follows:

$$
\begin{aligned}
& \lambda_{1}^{(0)}=0.9, \lambda_{2}^{(0)}=0.87, \lambda_{3}^{(0)}=0.92, \lambda_{4}^{(0)}=0.92, \\
& \lambda_{5}^{(0)}=0.9 \text { and } c_{0}=28.7 .
\end{aligned}
$$

Step 3. Using model (M-3), we establish the following optimization model:

$$
\begin{array}{ll} 
& \max J=\lambda_{1}+\lambda_{2}+\lambda_{3}+\lambda_{4}+\lambda_{5} \\
\text { s.t. } & \left(2 w_{1}+3 w_{2}+4\right) / 6.2 \geq \lambda_{1} \geq 0.9, \\
& \left(3 w_{1}+4 w_{2}-2 w_{3}+4\right) / 6.5 \geq \lambda_{2} \geq 0.87, \\
& \left(3 w_{2}+2 w_{3}+4\right) / 6 \geq \lambda_{3} \geq 0.92, \\
& \left(4 w_{1}-w_{2}+2 w_{3}+4\right) / 6.4 \geq \lambda_{4} \geq 0.92, \\
& \left(2 w_{1}+3 w_{2}+w_{3}+4\right) / 6.6 \geq \lambda_{5} \geq 0.9, \\
& 11 w_{1}+12 w_{2}+3 w_{3}+20 \geq 28.7, \\
& 0.25 \leq w_{1} \leq 0.4,0.15 \leq w_{2} \leq 0.3, w_{3}>w_{2}, \\
& w_{1}+w_{2}+w_{3}=1, w_{j} \geq 0, j=1,2,3 .
\end{array}
$$

Solving this model, we get the attribute weight vector:

$$
w^{(1)}=(0.388,0.296,0.316)^{T}
$$

and calculate the alternative comprehensive scale $c\left(w^{(1)}\right)$, and also the achievement scale $\mu\left(z_{j}\left(w^{(1)}\right)\right)(j=1$, $2, \ldots, 5)$.

$$
\begin{aligned}
& c\left(w^{(1)}\right)=28.768, \mu\left(z_{1}\left(w^{(1)}\right)\right)=0.9135, \\
& \mu\left(z_{2}\left(w^{(1)}\right)\right)=0.8794, \mu\left(z_{3}\left(w^{(1)}\right)\right)=0.92,
\end{aligned}
$$


$\mu\left(z_{4}\left(w^{(1)}\right)\right)=0.92, \mu\left(z_{5}\left(w^{(1)}\right)\right)=0.9061$.

Again, the decision maker is not satisfied with the result, then he gives the new lower bounds $\lambda_{j}^{(1)}(j=1$, $2, \ldots, 5)$ of the achievement scale of the alternatives $x_{j}$ $(j=1, \ldots, 5)$ according to the achievement scale $\mu\left(z_{j}\left(w^{(1)}\right)\right)$ $(j=1,2, \ldots, 5)$, and gives the new lower bound of the comprehensive scale $c_{1}$ as follows:

$\lambda_{1}^{(1)}=0.9, \lambda_{2}^{(1)}=0.86, \lambda_{3}^{(1)}=0.93, \lambda_{4}^{(1)}=0.9$,

$\lambda_{5}^{(1)}=0.9$ and $c_{1}=28.5$.

Using model (M-3), we establish the following optimization model:

$$
\max J=\lambda_{1}+\lambda_{2}+\lambda_{3}+\lambda_{4}+\lambda_{5}
$$

s.t. $\left(2 w_{1}+3 w_{2}+4\right) / 6.2 \geq \lambda_{1} \geq 0.9$,

$\left(3 w_{1}+4 w_{2}-2 w_{3}+4\right) / 6.5 \geq \lambda_{2} \geq 0.86$,

$\left(3 w_{2}+2 w_{3}+4\right) / 6 \geq \lambda_{3} \geq 0.93$,

$\left(4 w_{1}-w_{2}+2 w_{3}+4\right) / 6.4 \geq \lambda_{4} \geq 0.9$,

$\left(2 w_{1}+3 w_{2}+w_{3}+4\right) / 6.6 \geq \lambda_{5} \geq 0.9$,

$11 w_{1}+12 w_{2}+3 w_{3}+20 \geq 28.5$,

$0.25 \leq w_{1} \leq 0.4,0.15 \leq w_{2} \leq 0.3, w_{3}>w_{2}$,

$w_{1}+w_{2}+w_{3}=1, w_{j} \geq 0, j=1,2,3$.

Solving this model, we obtain the attributes weight vector:

$$
w^{(2)}=(0.36,0.3,0.34)^{T},
$$

then calculate the alternative comprehensive scale $c\left(w^{(2)}\right)$, and also the achievement scale $\mu\left(z_{j}\left(w^{(2)}\right)\right) \quad(j=1$, $2, \ldots, 5)$.

$$
\begin{aligned}
& c\left(w^{(2)}\right)=28.58, \mu\left(z_{1}\left(w^{(2)}\right)\right)=0.9065, \\
& \mu\left(z_{2}\left(w^{(2)}\right)\right)=0.8615, \mu\left(z_{3}\left(w^{(2)}\right)\right)=0.93, \\
& \mu\left(z_{4}\left(w^{(2)}\right)\right)=0.9094, \mu\left(z_{5}\left(w^{(2)}\right)\right)=0.903 .
\end{aligned}
$$

Step 4. The decision maker is satisfied with this result. Therefore, we can calculate the overall values $z_{j}\left(w^{(2)}\right)(j=1,2, \ldots, 5)$ of the alternatives $x_{j}(j=1,2, \ldots, 5)$ using Eq.(3)

$$
\begin{aligned}
& z_{1}\left(w^{(2)}\right)=\left(s_{6},-0.38\right), z_{2}\left(w^{(2)}\right)=\left(s_{6},-0.4\right), \\
& z_{3}\left(w^{(2)}\right)=\left(s_{6},-0.42\right), z_{4}\left(w^{(2)}\right)=\left(s_{6},-0.18\right), \\
& z_{5}\left(w^{(2)}\right)=\left(s_{6},-0.04\right) .
\end{aligned}
$$

and rank all alternatives according to the values of $z_{j}\left(w^{(2)}\right)(j=1,2, \ldots, 5)$.

$$
x_{5} \succ x_{4} \succ x_{1} \succ x_{2} \succ x_{3} \text {. }
$$

Hence, the most desirable candidate is $x_{5}$.

From the above example, we can see that the higher comprehensive scale, it will not always be the higher achievement scale of each alternative, so the DM will not be satisfied with the result. On the other hand, if we only use the achievement scale, it can not achieve the equivalent between the different alternatives effectively.
As a result, taking into account the two indexes, it will not only consider the single alternative's request, but also achieve the equivalent between the different alternatives effectively.

In Ref. 32, Xu ranked all the alternatives as $x_{5} \succ x_{4}$ $\succ x_{2} \succ x_{1} \succ x_{3}$. There is minor difference between ours. But both our method and Xu's method obtain the most desirable candidate is $x_{5}$. In Xu's model, it only considers the achievement scale (he called satisfactory degree) of each alternative. The method does not consider the comprehensive scale of all the alternatives. Furthermore, the overall values of Xu's method are expressed in virtual linguistic, and the virtual linguistic computed by the linguistic index, although they could rank the alternatives, the final virtual linguistic without syntax either semantics limits the interpretability. In our model, we not only consider the achievement scale, but the comprehensive scale. In the interactive process, the decision maker can know the satisfactory degree of each alternative, and also know the comprehensive degree of all the alternatives. The interactive process can be realized by giving and revising the achievement scale and comprehensive scale till an optimum satisfactory solution is achieved. Our final results are in 2-tuple linguistic form. It seems more reasonable that linguistic decision making should be in linguistic information.

\section{Concluding Remarks}

In this paper, we investigate the MADM problems, in which the attribute values are expressed in linguistic variables because of time pressure, lack of knowledge, and his/her limited information processing capabilities and the information about attribute weights is partly known because that: (1) a decision should be made under time pressure and lack of data, (2) many of the attributes are intangible or non-monetary because they reflect social and environmental impacts, and (3) a DM has limited attention and information processing capabilities. In the paper, we have defined some new concepts, such as the distance between two 2-tuple linguistic variables. We also define the expectation level of alternative, the achievement scale, the alternative comprehensive scale under linguistic settings. Based on these concepts, we have established an interactive procedure for solving the problem. The interactive process can be realized by giving and revising the achievement scale and comprehensive scale. The method not only uses the objective information adequately, but also considers the DM's interactive request in the maximum extend. Finally, the method has been applied to the evaluation of university faculty for tenure and promotion. The 
theoretical analysis and the computational results have showed robust method for solving the MADM problems with linguistic informati- on. In the real world, the decision process is made not only by one DM, but also is made by a group DMs, or the decision information is expressed in the form of uncertain linguistic variables $^{22,23,34}$, and the interactive procedure for multiple attribute group decision making under uncertain linguistic environment will be left for future work.

\section{Acknowledgements}

The author is very grateful to the associated editor and two anonymous referees for their insightful and constructive comments and suggestions that have led to an improved version of this paper. This work was partly supported by the National Natural Science Foundation of China (No. 90924027, No.71101043), National Basic Research Program of China (973 Program, No. 2010C B951104), Key Program of National Social Science Foundation of China (No.10AJY005), College Philosophy and Social Science Research Project of Jiangsu Province under Grant 2011SJD630007.

\section{References}

1. G. Bordogna, M. Fedrizzi, G. Pasi, A linguistic modeling of consensus in group decision making based on OWA operators, IEEE Transactions on Systems, Man, and Cybernetics-Part A: Systems and Humans 27 (1997) 126132.

2. J. Cheng, S. Lin, An interactive neural network based approach for solving multiple criteria decision making problems, Decision Support Systems 36 (2003) 137-146.

3. F. Chiclana, F. Herrera, E. Herrera-Viedma, Integrating three representation models in fuzzy multipurpose decision making based on fuzzy preference relations, Fuzzy Sets and Systems 97 (1998) 33-48.

4. Y.C. Dong, Y.F. Xu, S. Yu, Computing the numerical scale of the linguistic term set for the 2-tuple fuzzy linguistic representation model, IEEE Transactions on Fuzzy Systems 17 (2009) 1366-1378.

5. F. Herrera, S. Alonso, F. Chiclana, E. Herrera-Viedma, Computing with words in decision making: Foundations, trends and prospects, Fuzzy Optimization and Decision Making 8 (2009) 337-364.

6. F. Herrera, E. Herrera-Viedma, Linguistic decision analysis: steps for solving decision problems under linguistic information, Fuzzy Sets and Systems 115 (2000) 67-82.

7. F. Herrera, E. Herrera-Viedma, L. Martínez, A fusion approach for managing multi-granularity linguistic term sets in decision making, Fuzzy Sets and Systems 114 (2000) 43-58.

8. F. Herrera, L. Martínez, A 2-tuple fuzzy linguistic representation model for computing with words, IEEE Transactions on Fuzzy Systems 8 (2000) 746-752.

9. F. Herrera, L. Martínez, A model based on linguistic 2tuples for dealing with multigranular hierarchical linguistic contexts in multi-expert decision-making, IEEE Transactions on Sysetms, Man, and Cybernetics-Part B. Cybernetics 31 (2001) 227-234.

10. F. Herrera, L. Martínez, P.J. Sánchez, Managing nonhomogeneous information in group decision making, European Journal of Operational Research 166 (2005) 115-132.

11. S.H. Jiang, N.R. Xu, Interactive multi-objective decisionmaking method based on objective achievement scale and objective comprehensive scale, Systems EngineeringTheory \& Practice 19 (1999) 9-14.

12. S.H. Kim, B.S. Ahn, Interactive group decision making procedure under incomplete information, European Journal of Operational Research 116 (1999) 498-507.

13. S.H. Kim, S.H. Choi, J.K. Kim, An interactive procedure for multiple attribute group decision making with incomplete information: Range-based approach, European Journal of Operational Research 118 (1999) 139-152.

14. S.H. Kim, C.H. Han, An interactive procedure for multiattribute group decision making with incomplete information, Computers \& Operations Research 26 (1999) 755-772.

15. L. Levrat, A. Voisin, S. Bombardier, J. Bremont, Subjective evaluation of car seat comfort with fuzzy set techniques, International Journal of Intelligent Systems 12 (1997) 891-913.

16. L. Martínez, F. Herrera, An overview on the 2-tuple linguistic model for Computing with Words in Decision Making: Extensions, applications and challenges, Information Sciences 207 (2012) 1-18.

17. L. Martínez, D. Ruan, F. Herrera, Computing with words in decision support systems: An overiew on models and applications, International Journal of Computational Intelligence Systems 3 (2010) 382-395.

18. J.M. Mendel, Historical reflections and new positions on perceptual computing, Fuzzy Optimization and Decision Making 8 (2009) 325-335.

19. K.S. Park, S.H. Kim, Tools for interactive multi-attribute decision making with incompletely identified information, European Journal of Operational Research 98 (1997) 111-123.

20. M. Roubens, Fuzzy sets and decision analysis, Fuzzy Sets and Systems 90 (1997) 199-206.

21. J.H. Wang, J.Y. Hao, A new version of 2-tuple fuzzy linguistic representation model for computing with words, IEEE Transactions on Fuzzy Systems 14 (2006) 435-445.

22. Y.J. Xu, Z.J. Cai, Standard deviation method for determining the weights of group multiple attribute decision making under uncertain linguistic environment, Proceedings of the 7th World Congress on Intelligent Control and Automation, Chongqing,China, 2008, pp. 8311-8316.

23. Y.J. Xu, Q.L. Da, A method for multiple attribute decision making with incomplete weight information under 
uncertain linguistic environment, Knowledge-Based Systems 21 (2008) 837-841.

24. Y.J. Xu, Q.L. Da, Standard and mean deviation methods for linguistic group decision making and their applications, Expert Systems with Applications 37 (2010) 5905-5912.

25. Y.J. Xu, Q.L. Da, X.W. Liu, Some properties of linguistic preference relation and its ranking in group decision making, Journal of Systems Engineering and Electronics 21 (2010) 244-249.

26. Y.J. Xu, H.M. Wang, Appoaches based on 2-tuple linguistic power aggregation operators for multiple attribute group decision making under linguistic environment, Applied Soft Computing 11 (2011) 39883997.

27. Y.J. Xu, H.M. Wang, Distance measure for linguistic decision making, Systems Engineering Procedia 1 (2011) 450-456.

28. Y.J. Xu, H.M. Wang, Power geometric operators for group decision making under multiplicative linguistic preference relations, International Journal of Uncertainty, Fuzziness and Knowledge-Based Systems 20 (2012) 139-159.

29. Z.S. Xu, Deviation measures of linguistic preference relations in group decision making, Omega 33 (2005) 249254.

30. Z.S. Xu, An interactive approach to multiple attribute group decision making with multigranular uncertain linguistic information, Group Decision and Negotiation 18 (2009) 119-145.

31. Z.S. Xu, Interactive method based on alternative achievement scale and alternative comprehensive scale for multiple attribute decision making problems, Control and Decision 168 (2002) 171-184.

32. Z.S. Xu, An interactive procedure for linguistic multiple attribute decision making with incomplete weight information, Fuzzy Optimization and Decision Making 6 (2007) $17-27$.

33. Z.S. Xu, A method for multiple attribute decision making with incomplete weight information in linguistic setting, Knowledge-Based Systems 20 (2007) 719-725.

34. Z.S. Xu, Uncertain linguistic aggregation operators based approach to multiple attribute group decision making under uncertain linguistic environment, Information Sciences 168 (2004) 171-184.

35. R.R. Yager, On the retranslation process in Zadeh's paradigm of computing with words, IEEE Transactions on Systems, Man, and Cybernetics - Part B: Cybernetics 34 (2004) 1184-1195.

36. L.A. Zadeh, The concept of a linguistic variable and its application to approximate reasoning, Information Sciences 8 (1975) 199-249. 